\title{
TORONTO CLINICAL NEUROPATHY SCORE AND MODIFIED TORONTO CLINICAL NEUROPATHY SCORE DIAGNOSTIC TESTS IN DISTAL DIABETIC SENSORIMOTOR POLYNEUROPATHY PATIENTS
}

\author{
Running Head: Toronto Clinical NeuropathyScore \\ ${ }^{* 1}$ Mudjiani Basuki, ${ }^{1}$ Muhammad Hamdan, ${ }^{1}$ Fidiana, Fadil, ${ }^{1}$ Noormainiwati
}

ABSTRACT---Background: Distal diabetic sensorimotor polyneuropathy is a common complication occurred in diabetes mellituspatients. Even though the number of diabetes mellitus patients has been increasing, the prevalence is still far from its actual numbers due to diagnostic and method criteria.

Objectives: We aimed to determine Toronto Clinical Neuropathy (TCN) and modified Toronto clinical neuropathy $(m T C N)$ scores in distal diabetic sensorimotor polyneuropathy patients.

Methods: Cross-sectional study was carried out from October 29 ${ }^{\text {th }}$,2014-June $1^{\text {st }}, 2015$ in 77 diabetes mellitus patients who visited Outpatient Clinic. Polyneuropathy diagnosis was based on TCN and mTCN scores, with gold standard of peroneal and/or sural nerve conduction velocity examination.

Results: Area under curvevalue of TCN score was 84.5\% (95\% CI: 74.7\%-94.3\%). TCN diagnostic score above 4 had sensitivity, specificity, and accuracy of 96\%, 40\%, 82\%, respectively. On the other hand, TCN diagnostic score above 8 had sensitivity, specificity, accuracy of 72\%, 80\%, 74\%, respectively. The AUC value of mTCN score was 81.9\% (95\% CI: 71.8\%-92.1\%). mTCN diagnostic score above 8 had sensitivity, specificity, accuracy of 63\%, 75\%, $66 \%$, respectively.

Conclusion: TNC score above 4 could be used as a screening test, while score above 8 could be used as a diagnostic test for distal DSP.

Keywords---diagnostic test,distal diabetic sensorimotor polyneuropathy, mTCN score, TCN score.

\section{INTRODUCTION}

Diabetes mellitus (DM) is a group of metabolic disorders characterized by hyperglycemia as a result of impaired insulin secretion and activity.[1] 171 million people were estimated to suffer from this disease in 2000, and this number would increase to 366 million cases in 2030.[2, 3] The Indonesian Central Bureau of Statistics reported 133 million DM patients aged over 20 in 2003, with an estimated of 8.2 million and 5.5 million DM patients living in urban and rural areas, respectively.

\footnotetext{
${ }^{1}$ 1Neurology Department,Dr. Soetomo Teaching Hospital, Faculty of Medicine, Universitas Airlangga, Surabaya 60285, Indonesia Corresponding Authors: anibasuki@yahoo.com
} 
International Journal of Psychosocial Rehabilitation, Vol. 24, Issue 02, 2020

ISSN: $1475-7192$

Diabetic neuropathy is a group of clinical syndromes affecting various neuro system, either singular or combination. The symptoms and complaints are not specific with slow progression.[4] Diabetic peripheral neuropathy (DPN) or Distal sensorimotor polyneuropathy (DSP) or Distal symmetrical polyneuropathy (DSPN) relates to peripheral nervous system and becomes a common neuropathy type occurred in DM patients. Peripheral nervous system disorder can cause protective sensation loss and impaired perception ability, particularly diabetic ulcers in the legs. DSP is a major risk factor of amputation, and significantly can cause high morbidity in DM.[5]

DSP prevalence in the United States was around 50\% in chronic diabetes patients, 7 million populations with diabetic neuropathy and 2.7 million populations suffered from painful neuropathy.[4, 6]A study found 30\% neuropathy prevalence in DM patients.[7] DSP condition can affect patient's life quality, both mentally and physically. DSP correlates with numbness that commonly triggers balance disorder which subsequently causes patient to easily fall down. Neuropathy is one of major three falling risk factors, particularly in DM patients, in addition to retinopathy and vestibular dysfunction. Patient with severe DSP has ulceration and amputation risks. Around 50-70\% become the major cause of non-traumatic amputation. Diabetic DSP can decrease life quality, particularly pain complaints. Neuropathy pain is one of the most common disability symptoms in DSP patients. This symptom is difficult to treat and causes social distress and burden. This eventually results in higher treatment costs. In the U.S., a quarter of the total costs of diabetes care is spent on neuropathic treatment.[7]

Nerve biopsy or Electromyography (EMG) is a gold standard in making polyneuropathy diagnosis. EMG is an invasive diagnostic method that requires special tools and skills, is relatively expensive and sometimes causes patient's discomfort. A study found that combination of neuropathy symptoms, physical examination and abnormal EMG had a more accurate diagnostic score to make establish distal polyneuropathy.[8]

Toronto clinical neuropathy score (TCNS) was validated as a score in monitoring and diagnosing DSP.[9] TCNS scoring has a considerably high sensitivity and specificity. A study reported that TCNS had sensitivity $77.2 \%$ and specificity $75.6 \%$.[10] TCNS is quite valid since it combines DSP symptom and physical examination, including sensory evaluation (pain, temperature, touch, vibration and position), reflex physiology (patellar and Achilles reflexes) and symptoms (feet, legs and ataxia symptoms).[10] Modified TCNS since TCNS results varied greatly between assessors, age and body weight. Therefore, it must be generalized in order to obtain results that can increase scoring sensitivity in early DSP diagnosis.[11]We were interested in determining if mTCNShad a better diagnostic score than TCNS in diagnosing diabetic DSP compared to EMG as the gold standard.

\section{METHODS}

There were 77 subjects fulfilled DM type 2 criteria with DSP diagnosis who visited Endocrine Outpatient Unit of Dr. Soetomo Teaching Hospital, Surabaya, Indonesia, from October 2014-June 2015. The inclusion criteria wereDM type 2 subjects with DSP diagnosis aged 20-60 who signed informed consent. The study protocol was approved by the ethics committees of Dr. Soetomo Teaching Hospital, Surabaya, Indonesia.

It is a diagnostic test study to determine diagnostic score (sensitivity, specificity, Positive predictive value (PPV), Negative predictive value (NPV), accuracy and Likelihood ratio (LR) of TCNS and mTCNS to have early diagnose of DSP in DM patients compared to the gold standard of EMG examination. Data of score comparison was analyzed using $2 \times 2$ contingency table to obtain sensitivity, specificity, PPV and NPV in each confidence interval and LR value. 
International Journal of Psychosocial Rehabilitation, Vol. 24, Issue 02, 2020

ISSN: $1475-7192$

\section{RESULTS}

Demographic data

There were 77 DM subjects with DSP diagnosis, consisting of 27 males (35.1\%) and 50 females (64.9\%; Table 1). The subjects' average age was 51.90 5.619 (Table 2). The largest sample distribution belonged to age group of 51-60 with 45 subjects (58.4\%), followed by age group of $41-50$ with 29 subjects (37.7\%) and 3 subjects in the age group of 31-40 (3.9\%; Table 1).

\section{Clinical data}

We measured height, body weight, Body mass index (BMI), Peripheral neuropathy (PNP) symptom onset, DM onset and hypertension history. The subjects' average height was $157.23 \pm 7.287 \mathrm{~cm}$, with the tallest and the shortest subjects were $181 \mathrm{~cm}$ and $145 \mathrm{~cm}$, respectively (Table 3). The subjects' average weight was $61.17 \pm 9.908 \mathrm{~kg}$, with the biggest and the lowest weight were $100 \mathrm{~kg}$ and $43 \mathrm{~kg}$, respectively (Table 4). There were 32 overweight subjects (42\%), 4 underweight subjects (5\%) and 41 normal subjects (53\%; Table 1).

Most subjects had polyneuropathy symptom in 0-12 months onset (54 subjects, 70\%), followed by 12 subjects with $>12-24$-month onset (16\%) and 11 subjects with >24-month onset (14\%; Table 1). Most subjects suffered from DM with 0-5-year duration (45 subjects, 58.4\%), followed by 18 subjects with >5-10-year duration (23.4\%), 11 subjects with $>10-20$-year duration (14.3\%) and 3 subjects with $>20$-year duration (3.9\%; Table 1). There were 33 subjects with hypertension history (42.9\%) and 44 subjects without hypertension history (57.1\%; Table 1).

\section{EMG examination}

There were 57 subjects with EMG polyneuropathy (74\%) and 20 subjects without PNP (26\%; Table 1)

\section{TCNSdiagnostic score onEMG}

TCNS has cut-off point of 4 . Score 0-4 indicates normal condition, while score above 4 indicates neuropathy symptoms[9]. There were 67 PNP DM subjects with TCNS cut-off point 4 (87\%) and 10 subjects with normal cut-off point (13\%; Table 1).

Cross-tabulation between TCNS cut-off point 4 and EMG results showed similar diagnosis: 67 patients with PNP DM diagnosis measured by TCNS and 55 patients with positive PNP DM diagnosis measured by EMG. Of 10 subjects with normal diagnosis measured using TCNS, 8 subjects ( $80 \%)$ were diagnosed not having PNP measured using EMG (Table 5). The diagnosis score of TCNS cut-off point 4 was as follows: sensitivity 96\% (55/57, 95\% CI: 92\%-100\%); specificity40\% (8/20, 95\% CI: 19\%-61\%); PPV 82\% (55/67, 95\% CI: 73\%-91\%); NPV 80\% (8/10, 95\% CI: $55 \%-100 \%) ;$ prevalence $74 \%(57 / 77) ; \mathrm{LR}+=96 /(1-40)=1.61 ; \mathrm{LR}-=(1-96) / 40=0.09 ;$ accuracy $81.8 \%$ $[(55+8) / 77 \times 100 \%]$.

Cut-off point refers to as a boundary value between normal and abnormal, or between positive and negative test results. Cut-off point determination includes a bargaining process since increased sensitivity will cause decreased specificity in the similar data, and vice versa. Receiver operating curve (ROC) is a way to determine diagnostic test's cut-off point. It describes a bargaining process between sensitivity and specificity in the form of a graph. Sensitivity and specificity are represented on $y$-axis and $x$-axis, respectively. The higher the sensitivity, the lower the specificity, and vice versa. The closer the ROC to the diagonal line, the worse the results. The best cut-off point is the farthest point on the upper left of diagonal line. 
International Journal of Psychosocial Rehabilitation, Vol. 24, Issue 02, 2020

ISSN: $1475-7192$

ROC line of TNCS was far from the diagonal line, with the area under curve (AUC) value of $84.5 \%$ (95\%CI: 74.7\%-94.3\%. ROC procedure also obtained some alternative cut-off points of TCNS along with sensitivity value. Specificity value was calculated using Microsoft Excel. The software helped to find optimal cut-off point that was obtained from the intersection of sensitivity and specificity curves. The point was between score 7.5 and 8.5 Nevertheless, this scoring had no decimal score, therefore we took optimal value of 8 .

There were 41 subjects with TCNS cut-off point 8 who suffered from PNP DM (58.4\%) and 32 normal subjects (41.6\%; Table 1). Cross-tabulation between TCNS cut-off point 8 and EMG results showed similar diagnosis: 45 patients with PNP DM diagnosis measured using TCNS, 41 patients with positive PNP DM diagnosis measured using EMG (91.1\%). Of 32 subjects with normal diagnosis measured using TCNS, 16 subjects (50\%) were diagnosed not having PNP measured using EMG (Table 6). The diagnosis score of TCNS cut-off point 8 was as follows: Sensitivity $72 \%$ (41/57, 95\% CI: 60\%-84\%); specificity 80\% (16/20, 95\% CI: 62\%-98\%); PPV 91\% (41/45, 95\% CI: 83\%-99\%); NPV 50\% (16/32, 95\% CI: 33\%-67\%); prevalence 74\% (57/77); LR+ = 72/(1-80) = 3.60; LR- $=(1-$ $72) / 80=0.35 ;$ accuracy $74 \%[(41+16) / 77 \times 100 \%]$.

\section{mTCNS diagnostic scoreonEMG}

ROC line of mTCNS was far from the diagonal line, with the AUC value of $81.9 \%$ (95\%CI: $71.8 \%-2.1 \%$. ROC procedure also obtained some alternative cut-off points of mTCNS along with sensitivity value, followed by specificity measurement using Microsoft Excel. The software helped to find optimal cut-off point that was obtained from the intersection of sensitivity and specificity curves. The point was between score 7.5 and 8.5 (Table 7). Nevertheless, this scoring did not have decimal score, therefore we took optimal value of 8 .

There were 41 subjects with mTCNS cut-off point 8 who suffered from PNP DM (53\%) and 36 normal subjects (47\%; Table 1). Cross-tabulation between mTCNS cut-off point 8 and EMG results showed similar diagnosis: 41 patients with PNP DM diagnosis measured using mTCNS, 36 patients with positive PNP DM diagnosis measured using EMG (87.8\%). Of 36 subjects with normal diagnosis measured using mTCNS, 15 subjects (41.6\%) were diagnosed not having PNP measured using EMG. The diagnostic score of mTCNS cut-off point 8 was as follows: sensitivity 63\% (36/57, 95\% CI: 51\%-76\%); specificity 75\% (15/20, 95\% CI: 56\%-94\%); PPV 88\% (36/41, 95\% CI: 78\%-98\%); NPV 42\% (15/36, 95\% CI: 26\%-58\%); prevalence 74\% (57/77); LR+ = 63/(1-75) = 2.53; LR- $=(1-$ $63) / 75=0.49$; accuracy66.2\% [(36+15)/77 x 100\%].

\section{DISCUSSION}

The subjects' average age was 51.9 \pm 5.619 , with most subjects belonged to age group of 51-60 (58.4\%). This finding was consistent with a study conducted in India found that diabetic neuropathy subjects' average age was 50.44 \pm 10.35 .[12] On the other hand, a study in Sri Lanka found subjects' average age was 62.1 \pm 10.8 .[5]

There were 77 subjects, consisting of 27 males (35.1\%) and 50 females (64.7\%). The study consistent with the Sri Lankan study that obtained samples consisting of $37.1 \%$ of male and $62.9 \%$ of females.[5] The largest sample distribution belonged to age group of 51-60 with 45 subjects (58.4\%), followed by age group of 41-50 with 29 subjects (37.7\%) and 3 subjects in the age group of 31-40 (3.9\%). It was consistent with a multi-center study conducted in the United Kingdom that reported diabetic neuropathy increased along with age.[13] 
International Journal of Psychosocial Rehabilitation, Vol. 24, Issue 02, 2020

ISSN: $1475-7192$

There were 32 overweight diabetic polyneuropathy subjects with BMI above 25 (42\%) and 33 diabetic polyneuropathy subjects with hypertension (43\%). There were 45 subjects with DM duration ranged from 0 to 5 years (59\%). This study consistent with a study that reported correlation between diabetic neuropathy and modified cardiovascular risk factor, such as BMI and hypertension.[14] A study reported that age, diabetes duration and overweight correlated with increased diabetic neuropathy prevalence.[15]

TCNS above 4 had sensitivity 96\%, specificity 40\%, PPV 84\%, NPV 80\%, LR+ 1.61, LR- 0.09 and accuracy $82 \%$. Sensitivity 96\% indicated TCNS cut-off 4 ability in determining DM polyneuropathy as much as $96 \%$. Specificity $40 \%$ indicated lower ability in determining the absence of polyneuropathy.PPV 84\% indicated that if TCNS cut-off 4 showed polyneuropathy, EMG results also possibly had polyneuropathy as much as $84 \%$. NPV $80 \%$ indicated that if TCNS cut-off 4 obtained normal value, EMG results also possibly had $80 \%$ normal value. Accuracy $82 \%$ indicated TCNS cut-off 4 conformity with EMG as much as $82 \%$.

TCNS above 8 had sensitivity 72\%, specificity 80\%, PPV 91\%, NPV 50\%, LR+ 3.60, LR- 0.35 and accuracy 74\%. Sensitivity $72 \%$ indicated TCNS cut-off 8 ability in determining DM polyneuropathy as much as $72 \%$. Specificity $80 \%$ indicated high ability in determining the absence of polyneuropathy. PPV $91 \%$ indicated that if TCNS cut-off point 8 showed polyneuropathy, EMG results also possibly showed polyneuropathy as much as $91 \%$. NPV $50 \%$ indicated that if TCNS cut-off point 8 showed normal value, EMG results also possibly showed normal value as much as $50 \%$. Accuracy $74 \%$ indicated TCNS cut-off 8 conformity with EMG as much as $74 \%$.

mTCNS score above 8 had sensitivity 63\%, specificity 75\%, PPV 88\%, NPV 42\%, LR+ 2.53, LR- 0.49 and accuracy $66 \%$. Sensitivity 63\% indicated mTCNS cut-off 8 ability in determining DM polyneuropathy as much as $63 \%$. Specificity $75 \%$ indicated high ability in determining the absence of polyneuropathy. PPV $88 \%$ indicated that if mTCNS cut-off 8 showed polyneuropathy, EMG results also possibly showed polyneuropathy as much as $88 \%$. NPV $42 \%$ indicated that if mTCNS cut-off 8 showed normal value, EMG results also possibly showed normal value as much as $42 \%$. Accuracy $66 \%$ indicated mTCNS cut-off 8 conformity with EMG as much as $66 \%$.

It could be inferred that TCNS cut-off 4 was more appropriate for screening test since it had high sensitivity value (96\%) and low specificity value (40\%). On the other hand, TCNS cut-off 8 (sensitivity $72 \%$, specificity $80 \%$ ) and mTCNS cut-off 8 (sensitivity 63\%, specificity 75\%) could be utilized as diagnostic tests since they had a higher specificity value. LR+ value obtained by TCNS cut-off 4 (1.61) indicated comparison between PNP DM patients with positive TCNS and non-PNP DM patients with positive TCNS proportion was 1.61. LR- value obtained by TCNS cut-off 4 (0.09) indicated comparison between PNP DM patients with negative TCNS and non-PNP DM patients with negative TCNS proportion was 0.09. A study reported that a strong positive diagnostic score gave LR score higher than 1 , while a strong negative strong gave LR score closer to 0 . On the other hand, a moderate score gave LR score around 1. The strongest positive diagnostic score was obtained by TCNS cut-off 8 (LR+ 3.60, LR- 0.35).

\section{CONCLUSION}

TCNS above 4 was more appropriate for screening test since it had high sensitivity value (96\%). TCNS above 8 could be utilized as diagnostic test since it had high specificity value (80\%). TCNS had higher diagnostic score than mTCNS. 
International Journal of Psychosocial Rehabilitation, Vol. 24, Issue 02, 2020

ISSN: $1475-7192$

\section{REFERENCES}

[1] Maharani, A.I., Hasmono, D., Diansyah, M.N.Use of anti-dyslipidemia in type 2 diabetes mellitus. Indian Journal of Public Health Research and Development. 2019;10(8), pp. 1970-1976

[2] Wild S, Roglic G, Green A, Sicree R and King H. Global Prevalence of Diabetes: Estimates for the year 2000 and Projections for 2030. Diabetes Care 2004; 27:1047 - 1053.

[3] Wulandari, I., Kusnanto, Nufus, S.H.Motivation affects self-efficacy greater than age, sex, and education in diabetic patients in west coast area of Java island. Indian Journal of Public Health Research and Development. 2019; 10(8), pp. 2803-2807

[4] Romadhon, P.Z., Sutjahjo A., Novida, H., Prajitno, J.H., Tjokroprawiro, A. HBA1C and plasma transforming growth factor-beta 1 in type-2 diabetes mellitus patients. New Armenian Medical Journal. 2019;13(1), pp. 69-73

[5] Katulanda P, Ranasinghe P, Jayawardena R, Constantine GR, Sheriff MHR and Matthews DR. The prevalence, patterns and predictors of diabetic peripheral neuropathy in a developing country. Diabetology \& Metabolic Syndrome 2012; 21:1-8.

[6] Shakher J and Stevens MJ. Diabetes, Metabolic Syndrome and Obesity: Targets and Therapy. The Management of Diabetic Polyneuropathies 2011; 4:289-305.

[7] Callaghan BC, Cheng HT, Stables CL, Smith AL and Feldman EL. Diabetic Neuropahy: Clinical Manifestations and Current Treatments. Lancet Neurol 2012; 11:521-534.

[8] England JD, Gronseth GS, Franklin G, Miller RG, Asbury AK, Carter GT and al e. Distal symmetric polyneuropathy: A definition for clinical research: Report of the American Academy of Neurology, the American Association of Electrodiagnostic Medicine, and the American Academy of Physical Medicine and rehabilitation. Neurology 2005; 64:199 - 207.

[9] Bril V and Perkins BA. Original Article: Validation of The Toronto Clinical Scoring System for Diabetic Polyneuropathy. Diabetes Care 2002; 25:2048-2052.

[10] Liu F, Mao JP and Yan X. Toronto Clinical Scoring system in diabetic peripheral neuropathy. Journal of Central South University, Medical Science 2008; 33:1137-1141.

[11] Bril V, Tomioka S, Buchanan RA and Perkins BA. Original Article: Complications Reliability and Validity of the Modified Toronto Clinical Neuropathy Score in Diabetic Sensorimotor Polyneuropathy. Diabetic Medicine 2009; 26:240-246.

[12] Dutta A, Naorem S, Singh P and Wangjam K. Prevalence of Peripheral Neuropathy in Newly Diagnosed Type 2 Diabetics. Int J Diab Dev 2005; 25:30-32.

[13] Young MJ, Boulton AJM, Macleod AF, Williams DRR and Sonksen PH. A multicentre study of the prevalence of diabetic peripheral neuropathy in the United Kingdom hospital clinic population. Diabetologia 1993; 36:150-154.

[14] Tesfaye S, Chaturvedi N, Eaton S, Ward JD, Manes C, Tirgoviste CI, Witte DR and Fuller JH. Vascular Risk Factors and Diabetic Neuropathy. N Eng J Med 2005; 52:341-350.

[15] Al SK, Althwabia I, Khaled MB and Haddad F. Prevalence of peripheral neuropathy among patients with diabetes mellitus at King Hussein Hospital, Amman, Jordan. Rawal Medical Journal 2013; 38:92-96. 
International Journal of Psychosocial Rehabilitation, Vol. 24, Issue 02, 2020

ISSN: $1475-7192$

[16] Luaibi, H.M., Alfarhani, B.F., Hammza, R.A. Comparative assessment of catechin and gallic acid content in different brands of black and green tea (2018) International Journal of Pharmaceutical Research, 10 (4), pp. 585-589. https://www.scopus.com/inward/record.uri?eid=2-s2.085062401505\&doi=10.31838\%2fijpr\%2f2018.10.04.102\&partnerID=40\&md5=9fdbb15176b3353b1e03813 e5be5f3fb

[17] Debarshi kar mahapatra, vivek asati, sanjay kumar bharti (2019) recent therapeutic progress of chalcone scaffold bearing compounds as prospective anti-gout candidates. Journal of Critical Reviews, 6 (1), 15. doi:10.22159/jcr.2019v6i1.31760

[18] Shukla SS, Saraf S, Saraf S. "Fundamental Aspect and Basic Concept of Siddha Medicines." Systematic Reviews in Pharmacy 2.1 (2011), 48-54. Print. doi:10.4103/0975-8453.83439 
International Journal of Psychosocial Rehabilitation, Vol. 24, Issue 02, 2020

ISSN: $1475-7192$

TABLES

Table 1.Subject's characteristics by sex

\begin{tabular}{|c|c|c|}
\hline Variables & $\mathrm{n}$ & percentage $(\%)$ \\
\hline \multicolumn{3}{|l|}{ Sex } \\
\hline Male & 27 & 35.1 \\
\hline Female & 65 & 64.9 \\
\hline Age group & 77 & 100.0 \\
\hline $31-40$ & 3 & 3.9 \\
\hline $41-50$ & 29 & 37.7 \\
\hline $51-60$ & 45 & 58.4 \\
\hline \multicolumn{3}{|l|}{ BMI } \\
\hline Underweight & 4 & 5.2 \\
\hline Normal & 41 & 53.2 \\
\hline Overweight & 32 & 41.6 \\
\hline \multicolumn{3}{|c|}{ Polyneuropathy onset } \\
\hline $0-12$ months & 54 & 70.1 \\
\hline$>12-24$ months & 12 & 15.6 \\
\hline$>24$ months & 11 & 14.3 \\
\hline \multicolumn{3}{|l|}{ DM duration } \\
\hline $0-5$ years & 45 & 58.4 \\
\hline$>5-10$ years & 18 & 23.4 \\
\hline$>10-20$ years & 11 & 14.3 \\
\hline$>20$ years & 3 & 3.9 \\
\hline \multicolumn{3}{|c|}{ Hypertension history } \\
\hline With & 33 & 42.9 \\
\hline Without & 44 & 57.1 \\
\hline \multicolumn{3}{|l|}{ EMG results } \\
\hline PNP & 57 & 57 \\
\hline Non-PNP & 20 & 26.0 \\
\hline \multicolumn{3}{|c|}{ TCNS cut-off point 4} \\
\hline PNP DM & 67 & 87 \\
\hline Normal & 10 & 13 \\
\hline \multicolumn{3}{|c|}{ TCNS cut-off point 8} \\
\hline PNP DM & 45 & 58.4 \\
\hline Normal & 32 & 41.6 \\
\hline \multicolumn{3}{|c|}{ mTCNS cut-off point 8} \\
\hline PNP DM & 41 & 53 \\
\hline Normal & 36 & 47 \\
\hline
\end{tabular}


International Journal of Psychosocial Rehabilitation, Vol. 24, Issue 02, 2020

ISSN: $1475-7192$

Table 2.Subjects' characteristics by average age

\begin{tabular}{clcc}
\hline & & Subjects & \\
\hline & Mean \pm SD & Minimum & Maximum \\
\hline Age (year) & $51.90 \pm 5.619$ & 36 & 60 \\
\hline
\end{tabular}

Table 3.Subjects' characteristics by height

\begin{tabular}{cccc}
\hline & & Subjects & \\
\hline & Mean \pm SD & Minimum & Maximum \\
\hline Height $(\mathbf{c m})$ & $157.23 \pm 7.287$ & 145 & 181 \\
\hline
\end{tabular}

Table4.Subjects' characteristics by weight

\begin{tabular}{cccc}
\hline & & Subjects & \\
& Mean \pm SD & Minimum & Maximum \\
\hline Weight $(\mathbf{k g})$ & $61.17 \pm 9.908$ & 43 & 100 \\
\hline
\end{tabular}

Table5.Cross-tabulation ( $2 \times 2$ table) of TCNS cut-offpoint 4 andEMG

\begin{tabular}{|c|c|c|c|c|}
\hline & & \multicolumn{3}{|c|}{ EMG } \\
\hline & & PNP DM & Non- & Total \\
\hline & & \multicolumn{3}{|c|}{ PNP } \\
\hline \multirow{6}{*}{$\begin{array}{l}\text { TCNS } \\
\text { cutoff point } 4\end{array}$} & & 55 & 12 & 67 \\
\hline & PNP DM & $82.1 \%$ & $17.9 \%$ & $100 \%$ \\
\hline & & 2 & 8 & 10 \\
\hline & Normal & $20 \%$ & $80 \%$ & $100 \%$ \\
\hline & & 57 & 20 & 77 \\
\hline & Total & $74 \%$ & $26 \%$ & $100 \%$ \\
\hline
\end{tabular}

Table6.Cross-tabulation ( $2 \times 2$ table) of TCNS cut-off point 8 andEMG

\begin{tabular}{lcccc}
\hline & & \multicolumn{3}{c}{ EMG } \\
\hline & & PNP DM & Non-PNP & Total \\
\hline & & 41 & 4 & 45 \\
TCNS & PNP DM & $91.1 \%$ & $8.9 \%$ & $100 \%$ \\
cutoff point 8 & & 16 & 16 & 32 \\
& Normal & $50 \%$ & $50 \%$ & $100 \%$ \\
& & 57 & 20 & 77 \\
& Total & $74 \%$ & $26 \%$ & $100 \%$ \\
\hline
\end{tabular}


International Journal of Psychosocial Rehabilitation, Vol. 24, Issue 02, 2020

ISSN: $1475-7192$

Table 7.mTCNS sensitivity and specificityfrom some alternative cut-points

\begin{tabular}{lccc}
\hline No. & Cut-off Point & Sensitivity & Specificity \\
\hline $\mathbf{1}$ & 1.00 & 1.00 & 0.00 \\
$\mathbf{2}$ & 2.50 & 1.00 & 0.05 \\
$\mathbf{3}$ & 3.50 & .98 & 0.30 \\
$\mathbf{4}$ & 4.50 & .93 & 0.50 \\
$\mathbf{5}$ & 5.50 & .84 & 0.50 \\
$\mathbf{6}$ & 6.50 & .81 & 0.60 \\
$\mathbf{7}$ & 7.50 & .77 & 0.70 \\
$\mathbf{8}$ & 8.50 & .63 & 0.75 \\
$\mathbf{9}$ & 9.50 & .58 & 0.90 \\
$\mathbf{1 0}$ & 10.50 & .40 & 0.95 \\
$\mathbf{1 1}$ & 11.50 & .33 & 1.00 \\
$\mathbf{1 2}$ & 12.50 & .30 & 1.00 \\
$\mathbf{1 3}$ & 13.50 & .25 & 1.00 \\
$\mathbf{1 4}$ & 14.50 & .21 & 1.00 \\
$\mathbf{1 5}$ & 15.50 & .18 & 1.00 \\
$\mathbf{1 6}$ & 16.50 & .16 & 1.00 \\
$\mathbf{1 7}$ & 17.50 & .14 & 1.00 \\
$\mathbf{1 8}$ & 18.50 & .12 & 1.00 \\
\end{tabular}

Table8.Cross-tabulation ( $2 \times 2$ table) of mTCNS cut-off point 8 and EMG

\begin{tabular}{lcccc}
\hline & & \multicolumn{3}{c}{ EMG } \\
\hline & & PNP DM & Non-PNP & Total \\
\hline mTCNS & & 36 & 5 & 41 \\
cutoff point 8 & PNP DM & $87.8 \%$ & $12.2 \%$ & $100 \%$ \\
& & 21 & 15 & 36 \\
& Normal & $58.4 \%$ & $41.6 \%$ & $100 \%$ \\
& & 57 & 20 & 77 \\
& Total & $74 \%$ & $26 \%$ & $100 \%$ \\
\hline
\end{tabular}

